\title{
Regularity properties of degenerate convolution-elliptic equations
}

\author{
Hummet K Musaev and Veli B Shakhmurov $2,3^{*}$
}

\author{
"Correspondence: \\ veli.sahmurov@okan.edu.tr \\ ${ }^{2}$ Department of Mechanical \\ Engineering, Okan University, Akfirat \\ Beldesi, Tuzla, Istanbul, 34959, \\ Turkey \\ ${ }^{3}$ Department of Mathematics, \\ Azerbaijan Khazar University, Baku, \\ Azerbaijan \\ Full list of author information is \\ available at the end of the article
}

\begin{abstract}
The coercive properties of degenerate abstract convolution-elliptic equations are investigated. Here we find sufficient conditions that guarantee the separability of these problems in $L_{p}$ spaces. It is established that the corresponding convolution-elliptic operator is positive and is also a generator of an analytic semigroup. Finally, these results are applied to obtain the maximal regularity properties of the Cauchy problem for a degenerate abstract parabolic equation in mixed $L_{\mathbf{p}}$ norms, boundary value problems for degenerate integro-differential equations, and infinite systems of degenerate elliptic integro-differential equations.
\end{abstract}

MSC: 34G10; 45J05; 45K05

Keywords: positive operators; abstract weighted spaces; operator-valued multipliers; boundary value problems; convolution equations; integro-differential equations

\section{Introduction, notations, and background}

In recent years, maximal regularity properties for differential operator equations, especially of parabolic and elliptic type, have been studied extensively e.g. in [1-14] and the references therein. Moreover, convolution-differential equations (CDEs) have been treated e.g. in [2, 15-19] (for comprehensive references see [18]). Convolution operators in Banach-valued spaces were studied e.g. in [8-10, 20-23]. However, the convolutiondifferential operator equations (CDOEs) are a relatively poorly investigated subject. In [2] the parabolic type CDEs with bounded operator coefficients were investigated. In [9] the regularity properties of degenerate ordinary CDOEs were studied. The main aim of the present paper is to study the following degenerate elliptic CDOEs:

$$
\sum_{|\alpha| \leq l} a_{\alpha} * D^{[\alpha]} u+(A+\lambda) * u=f(x)
$$

and the Cauchy problem for the degenerate parabolic CDOE

$$
\frac{\partial u}{\partial t}+\sum_{|\alpha| \leq l} a_{\alpha} * D^{[\alpha]} u+A * u=f(t, x), \quad u(0, x)=0,
$$

in $E$-valued $L_{p}$ spaces, where $E$ is a Banach space, $A=A(x)$ is a linear operator in $E$, $a_{\alpha}=$ $a_{\alpha}(x)$ are complex-valued functions, $\alpha=\left(\alpha_{1}, \alpha_{2}, \ldots, \alpha_{n}\right), \lambda$ is a complex number, $\gamma=\gamma(x)$

(c) 2016 Musaev and Shakhmurov. This article is distributed under the terms of the Creative Commons Attribution 4.0 International License (http://creativecommons.org/licenses/by/4.0/), which permits unrestricted use, distribution, and reproduction in any medium, provided you give appropriate credit to the original author(s) and the source, provide a link to the Creative Commons license, and indicate if changes were made. 
is a positive measurable function on $\Omega \subset R^{n}$, and

$$
D^{[\alpha]}=D_{x_{1}}^{\left[\alpha_{1}\right]} D_{x_{2}}^{\left[\alpha_{2}\right]} \cdots D_{x_{n}}^{\left[\alpha_{n}\right]}, \quad D_{x_{i}}^{\left[\alpha_{i}\right]}=\left(\gamma(x) \frac{\partial}{\partial x_{i}}\right)^{\alpha_{i}} .
$$

Here the convolutions $a_{\alpha} * D^{[\alpha]} u$ and $A * u$ are defined in the distribution sense (see e.g. [1]).

In applications, particularly the above equations describe the charged particle motion for certain configurations of oscillating magnetic fields (see e.g. [24]). Maximal regularity has proven very useful in handling some concrete non-linear evolution equations as shown by [25] and [23], which deal with the Navier-Stokes equations of fluid dynamics. One of the main features of the present work is that the convolution equations are degenerate on some points of $\mathbb{R}=(-\infty, \infty)$. Moreover, equation (1.1) has a variable operator coefficient. Since such a type equations occur in applications, it is important to show the existence and uniqueness of the solution. In this paper, we establish the separability properties of the problem (1.1) and the maximal regularity of Cauchy problem for parabolic CDOE. Moreover, we prove that the operator generated by problem (1.1) is positive. The main tools of this work are the operator-valued Fourier multipliers. Since equation (1.1) has unbounded operator coefficients there occurs some difficulty. This fact is derived by using the representation formula for a solution of the problem (1.1) and operator-valued multiplier in $L_{p, \gamma}\left(R^{n} ; E\right)$.

Let $E$ be a Banach space and $\gamma=\gamma(x), x=\left(x_{1}, x_{2}, \ldots, x_{n}\right)$ be a positive measurable weighted function on a measurable subset $\Omega \in R^{n}$. Let $L_{p, \gamma}(\Omega ; E)$ denote the space of strongly $E$-valued functions that are defined on $\Omega$ with the norm

$$
\|f\|_{L_{p, \gamma}}=\|f\|_{L_{p, \gamma}(\Omega ; E)}=\int_{\Omega}\left(\|f(x)\|_{E}^{p} \gamma(x) d x\right)^{1 / p}, \quad 1 \leq p<\infty .
$$

For $\gamma(x) \equiv 1$, the space $L_{p, \gamma}(\Omega, E)$ will be denoted by $L_{p}=L_{p}(\Omega ; E)$.

$$
\|f\|_{L_{\infty, p, \gamma}(\Omega ; E)}=\operatorname{ess} \sup _{x \in \Omega}\left[\gamma(x)\|f(x)\|_{E}\right]
$$

The weight $\gamma(x)$ we will consider to satisfy an $A_{p}$ condition; i.e., $\gamma(x) \in A_{p}, 1<p<\infty$, if there is a positive constant $C$ such that

$$
\left(\frac{1}{|Q|} \int_{Q} \gamma(x) d x\right)\left(\frac{1}{|Q|} \int_{Q} \gamma^{-\frac{1}{p-1}}(x) d x\right)^{p-1} \leq C
$$

for all compacts $Q \subset R^{n}$.

Let $\mathbb{C}$ be the set of complex numbers and

$$
S_{\varphi}=\{\lambda ; \lambda \in \mathbb{C},|\arg \lambda| \leq \varphi\} \cup\{0\}, \quad 0 \leq \varphi<\pi .
$$

Let $E_{1}$ and $E_{2}$ be two Banach spaces and let $B\left(E_{1}, E_{2}\right)$ denote the spaces of bounded linear operators acting from $E_{1}$ to $E_{2}$. For $E_{1}=E_{2}=E$ we denote $B(E, E)$ by $B(E)$.

A closed linear operator function $A=A(x)$ is said to be uniformly $\varphi$-positive in Banach space $E$, if $D(A(x))$ is dense in $E$ and does not depend on $x$ and there is a positive constant $M$ so that

$$
\left\|(A(x)+\lambda I)^{-1}\right\|_{B(E)} \leq M(1+|\lambda|)^{-1}
$$


for every $x \in R^{n}$ and $\lambda \in S_{\varphi}, \varphi \in[0, \pi)$, where $I$ is an identity operator in $E$. Sometimes instead of $A+\lambda I$ we will write $A+\lambda$ and it will be denoted by $A_{\lambda}$. It is well known [11], Section 1.14.1, that there exist fractional powers $A^{\theta}$ of the positive operator $A$.

Let $E\left(A^{\theta}\right)$ denote the space $D\left(A^{\theta}\right)$ with the graphical norm

$$
\|u\|_{E\left(A^{\theta}\right)}=\left(\|u\|^{p}+\left\|A^{\theta} u\right\|^{p}\right)^{\frac{1}{p}}, \quad 1 \leq p<\infty,-\infty<\theta<\infty .
$$

Let $S=S\left(R^{n} ; E\right)$ denote a Schwartz class, i.e. the space of $E$-valued rapidly decreasing smooth functions on $R^{n}$ equipped with its usual topology generated by seminorms. $S\left(R^{n} ; \mathbb{C}\right)$ is denoted by just $S$.

Let $S^{\prime}\left(R^{n} ; E\right)$ denote the space of all continuous linear operators, $L: S \rightarrow E$, equipped with the bounded convergence topology. Recall $S\left(R^{n} ; E\right)$ is norm dense in $L_{p, \gamma}\left(R^{n} ; E\right)$ when $1<p<\infty, \gamma \in A_{p}$.

Let $\Omega$ be a domain in $R^{n}$. $C(\Omega, E)$ and $C^{m}(\Omega ; E)$ will denote the spaces of $E$-valued bounded uniformly strongly continuous and $m$-times continuously differentiable functions on $\Omega$, respectively.

Let $\alpha=\left(\alpha_{1}, \alpha_{2}, \ldots, \alpha_{n}\right)$, where $\alpha_{i}$ are integers. An $E$-valued generalized function $D^{\alpha} f$ is called a generalized derivative in the sense of Schwartz distributions of the function $f \in$ $S\left(R^{n}, E\right)$, if the equality

$$
\left\langle D^{\alpha} f, \varphi\right\rangle=(-1)^{|\alpha|}\left\langle f, D^{\alpha} \varphi\right\rangle
$$

holds for all $\varphi \in S$.

Let $F$ denote the Fourier transform. Through this section the Fourier transformation of a function $f$ will be denoted by $\widehat{f}, F f=\check{f}$, and $F^{-1} f=\check{f}$. It is well known that

$$
F\left(D_{x}^{\alpha} f\right)=\left(i \xi_{1}\right)^{\alpha_{1}} \cdots\left(i \xi_{n}\right)^{\alpha_{n}} \widehat{f}, \quad D_{\xi}^{\alpha}(F(f))=F\left[\left(-i x_{1}\right)^{\alpha_{1}} \cdots\left(-i x_{n}\right)^{\alpha_{n}} f\right]
$$

for all $f \in S^{\prime}\left(R^{n} ; E\right)$.

Suppose $E_{1}$ and $E_{2}$ are two Banach spaces. $A$ function $\Psi \in L_{\infty}\left(R^{n} ; B\left(E_{1}, E_{2}\right)\right)$ is called a multiplier from $L_{p, \gamma}\left(R^{n} ; E_{1}\right)$ to $L_{p, \gamma}\left(R^{n} ; E_{2}\right)$ for $p \in(1, \infty)$ if the map $u \rightarrow T u=F^{-1} \Psi(\xi) F u$, $u \in S\left(R^{n} ; E_{1}\right)$ is well defined and extends to a bounded linear operator

$$
T: L_{p, \gamma}\left(R^{n} ; E_{1}\right) \rightarrow L_{p, \gamma}\left(R^{n} ; E_{2}\right) .
$$

The space of all Fourier multipliers from $L_{p, \gamma}\left(R^{n} ; E_{1}\right)$ to $L_{p, \gamma}\left(R^{n} ; E_{2}\right)$ will be denoted by $M_{p, \gamma}^{p, \gamma}\left(E_{1}, E_{2}\right)$. For $E_{1}=E_{2}=E$ we denote $M_{p, \gamma}^{p, \gamma}\left(E_{1}, E_{2}\right)$ by $M_{p, \gamma}^{p, \gamma}(E)$.

A Banach space $E$ is called a $U M D$-space $[26,27]$ if the Hilbert operator

$$
(H f)(x)=\lim _{\varepsilon \rightarrow 0} \int_{|x-y|>\varepsilon} \frac{f(y)}{x-y} d y
$$

is bounded in $L_{p}(R ; E), p \in(1, \infty)$ (see e.g. [4]). UMD spaces include e.g. $L_{p}, l_{p}$ spaces and the Lorentz spaces $L_{p q}, p, q \in(1, \infty)$. 
A set $K \subset B\left(E_{1}, E_{2}\right)$ is called $R$-bounded (see $[4,5,15,28]$ ) if there is a constant $C>0$ such that, for all $T_{1}, T_{2}, \ldots, T_{m} \in K$ and $u_{1}, u_{2}, \ldots, u_{m} \in E_{1}, m \in N$

$$
\int_{0}^{1}\left\|\sum_{j=1}^{m} r_{j}(y) T_{j} u_{j}\right\|_{E_{2}} d y \leq C \int_{0}^{1}\left\|\sum_{j=1}^{m} r_{j}(y) u_{j}\right\|_{E_{1}} d y,
$$

where $\left\{r_{j}\right\}$ is a sequence of independent symmetric $\{-1 ; 1\}$-valued random variables on $[0,1]$ and $\mathbb{N}$ denotes the set of natural numbers. The smallest $C$ for which the above estimate holds is called the $R$-bound of $K$ and is denoted by $R(K)$.

Definition 1.1 A Banach space $E$ is said to be a space satisfying the weighted multiplier condition if for any $\Psi \in C^{(n)}\left(R^{n} \backslash\{0\} ; B(E)\right)$ the $R$-boundedness of the set $\left\{|\xi|^{|\beta|} D_{\xi}^{\beta} \Psi(\xi): \xi \in\right.$ $\left.R^{n} \backslash\{0\}, \beta=\left(\beta_{1}, \beta_{2}, \ldots, \beta_{n}\right), \beta_{k} \in\{0,1\}\right\}$ implies that $\Psi$ is a Fourier multiplier in $L_{p, \gamma}\left(R^{n} ; E\right)$, i.e., $\Psi \in M_{p, \gamma}^{p, \gamma}(E)$ for any $p \in(1, \infty)$.

Remark 1.1 Note that, if $E$ is a $U M D$ space, then by virtue of $[5,6,13,21]$ it satisfies the multiplier condition.

Definition 1.2 A positive operator $A(x), x \in R^{n}$ is said to be uniformly $R$-positive in a Banach space $E$ if there exists a $\varphi=\varphi_{A} \in[0, \pi)$ such that the set $L_{A}=\left\{\xi(A+\xi)^{-1}: \xi \in S_{\varphi}\right\}$ is uniformly $R$-bounded, i.e.

$$
\sup _{x \in R^{n}} R\left(\left\{\left[A(x)(A(x)+\xi I)^{-1}\right]: \xi \in S_{\varphi}\right\}\right) \leq M .
$$

Note that in Hilbert spaces every norm bounded set is $R$-bounded. Therefore, in Hilbert spaces all positive operators are $R$-positive.

Let $h \in \mathbb{R}, m \in \mathbb{N}$, and $e_{k}, k=1,2, \ldots, n$, be standard unit vectors of $R^{n}$. Let

$$
\Delta_{k}(h) f(x)=f\left(x+h e_{k}\right)-f(x) .
$$

Let $A=A(x), x \in R^{n}$ be closed linear operator in $E$ with domain $D(A)$ independent of $x$. The Fourier transformation of $A(x)$ is a linear operator with the domain $D(A)$ defined as

$$
\hat{A} u(\varphi)=A u(\hat{\varphi}) \quad \text { for } u \in S^{\prime}\left(R^{n} ; E(A)\right), \varphi \in S\left(R^{n}\right) .
$$

(For details see [1].)

$A(x)$ is differentiable if we have the limit

$$
\left(\frac{\partial A}{\partial x_{k}}\right) u=\lim _{h \rightarrow 0} \frac{\Delta_{k}(h) A(x) u}{h}, \quad k=1,2, \ldots n, u \in D(A)
$$

in the sense of the $E$-norm.

Let $E_{0}$ and $E$ be two Banach spaces, where $E_{0}$ is continuously and densely embeds into $E$. Let $l$ be a integer number. $W_{p, \gamma}^{l}\left(R^{n} ; E_{0}, E\right)$ denotes the space of all functions from $S^{\prime}\left(R^{n} ; E_{0}\right)$ such that $u \in L_{p, \gamma}\left(R^{n} ; E_{0}\right)$ and the generalized derivatives $D_{k}^{l} u=\frac{\partial^{l} u}{\partial x_{k}^{l}} \in L_{p, \gamma}\left(R^{n} ; E\right)$ with the norm

$$
\|u\|_{W_{p, \gamma}^{l}\left(R^{n} ; E_{0}, E\right)}=\|u\|_{L_{p, \gamma}\left(R^{n} ; E_{0}\right)}+\sum_{k=1}^{n}\left\|D_{k}^{l} u\right\|_{L_{p, \gamma}\left(R^{n} ; E\right)}<\infty .
$$


It is clear that

$$
W_{p, \gamma}^{l}\left(R^{n} ; E_{0}, E\right)=W_{p, \gamma}^{l}\left(R^{n} ; E\right) \cap L_{p, \gamma}\left(R^{n} ; E_{0}\right) .
$$

Consider the problem

$$
\sum_{|\alpha| \leq l} a_{\alpha} * D^{\alpha} u+(A+\lambda) * u=f(x)
$$

where $A=A(x)$ is a linear operator in $E, a_{\alpha}=a_{\alpha}(x)$ are complex-valued functions, $\alpha=$ $\left(\alpha_{1}, \alpha_{2}, \ldots, \alpha_{n}\right), \lambda$ is a complex parameter.

A function $u \in W_{p, \gamma}^{l}\left(R^{n} ; E(A), E\right)$ satisfying equation (1.2) a.e. on $R^{n}$ is called the solution of equation (1.2).

The elliptic CDOE (1.2) is said to be uniform separable in $L_{p, \gamma}\left(R^{n} ; E\right)$ if equation (1.2) has a unique solution $u$ for $f \in L_{p, \gamma}\left(R^{n} ; E\right)$ and the following coercive estimate holds:

$$
\sum_{|\alpha| \leq l}\left\|a_{\alpha} * D^{\alpha} u\right\|_{L_{p, \gamma}\left(R^{n} ; E\right)}+\|A * u\|_{L_{p, \gamma}\left(R^{n} ; E\right)} \leq C\|f\|_{L_{p, \gamma}\left(R^{n} ; E\right)},
$$

where the positive constant $C$ is independent of $f$.

In a similar way to [3], Theorem $\mathrm{A}_{0}$, we obtain the following.

Proposition $\mathbf{A}_{0}$ Let $E$ be a UMD space and $\gamma \in A_{p}$. Assume $\Psi_{h}$ is a set of operator functions from $C^{n}\left(R^{n} \backslash\{0\} ; B(E)\right)$ depending on the parameter $h \in Q \in \mathbb{R}$ and there is a positive constant $K$ such that

$$
\sup _{h \in Q} R\left(\left\{|\xi|^{|\beta|} D^{\beta} \Psi_{h}(\xi): \xi \in R^{n} \backslash\{0\}, \beta_{k} \in\{0,1\}\right\}\right) \leq K .
$$

Then the set $\Psi_{h}$ is a uniformly bounded collection of Fourier multipliers in $L_{p, \gamma}\left(R^{n} ; E\right)$.

Let $E_{1}$ and $E_{2}$ be two Banach spaces. Suppose $T \in B\left(E_{1}, E_{2}\right)$ and $1 \leq p<\infty . \tilde{T} \in$ $B\left(L_{p}\left(R^{n} ; E_{1}\right), L_{p}\left(R^{n} ; E_{2}\right)\right)$ will denote the operator $(\tilde{T} f)(x)=T(f(x))$ for $f \in L_{p}\left(R^{n} ; E_{1}\right)$ and $x \in R^{n}$.

From [10] we have the following.

Proposition $\mathbf{A}_{1}$ Let $1 \leq p<\infty$. If $W \subset B\left(E_{1}, E_{2}\right)$ is R-bounded, then the collection $\tilde{W}=$ $\{\tilde{T}: T \in W\} \subset B\left(L_{p}\left(R^{n} ; E_{1}\right), L_{p}\left(R^{n} ; E_{2}\right)\right)$ is also R-bounded.

\section{Elliptic CDOE}

Condition 2.1 Assume $\hat{A}(\xi)$ is a uniformly positive operator in $E$ and $a_{\alpha} \in L_{\infty}\left(R^{n}\right)$ such that

$$
L(\xi)=\sum_{|\alpha| \leq l} \hat{a}_{\alpha}(\xi)(i \xi)^{\alpha} \in S_{\varphi_{1}}, \quad|L(\xi)| \geq C \sum_{k=1}^{n}\left|\hat{a}_{k}\right|\left|\xi_{k}\right|^{l},
$$

for

$$
k=k(\alpha)=\left(\alpha_{1}, \alpha_{2}, \ldots, \alpha_{n}\right), \quad \alpha_{k}=l, \alpha_{i}=0, i \neq k,
$$




$$
\varphi_{1} \in[0, \pi), \quad \xi=\left(\xi_{1}, \xi_{2}, \ldots, \xi_{n}\right) \in R^{n}
$$

where $a_{k}$ denote the coefficients of $\xi_{k}^{l}$ in the polynomial $L(\xi)$. Consider

$$
\begin{aligned}
& \sigma_{0}(\xi, \lambda)=\lambda D(\xi, \lambda), \quad \sigma_{1}(\xi, \lambda)=\hat{A}(\xi) D(\xi, \lambda) \\
& \sigma_{2}(\xi, \lambda)=\sum_{|\alpha| \leq l}|\lambda|^{1-\frac{|\alpha|}{l}} a_{\alpha}(\xi)(i \xi)^{\alpha} D(\xi, \lambda)
\end{aligned}
$$

where

$$
D(\xi, \lambda)=[\hat{A}(\xi)+L(\xi)+\lambda]^{-1}
$$

Lemma 2.1 Assume the Condition 2.1 holds and $\lambda \in S_{\varphi_{2}}$ with $\varphi_{2} \in[0, \pi)$, where $\varphi_{A}+\varphi_{1}+$ $\varphi_{2}<\pi$, then the operator functions $\sigma_{i}(\xi, \lambda)$ are uniformly bounded, i.e.,

$$
\left\|\sigma_{i}(\xi, \lambda)\right\|_{B(E)} \leq C, \quad i=0,1,2
$$

Proof By virtue of [4], Lemma 2.3, for $L(\xi) \in S_{\varphi_{1}}, \lambda \in S_{\varphi_{2}}$, and $\varphi_{1}+\varphi_{2}<\pi$, there is a positive constant $C$ such that

$$
|\lambda+L(\xi)| \geq C(|\lambda|+|L(\xi)|) .
$$

Since $L(\xi) \in S_{\varphi_{1}}$, in view of (2.1) and the resolvent properties of positive operators, we see that $\hat{A}(\xi)+L(\xi)+\lambda$ is invertible and

$$
1+M|\lambda+L(\xi)|(1+|\lambda+L(\xi)|)^{-1} \leq M_{1}
$$

Next, let us consider $\sigma_{2}$. It is clear that

$$
\left\|\sigma_{2}(\xi, \lambda)\right\|_{B(E)} \leq C \sum_{|\alpha| \leq l}|\lambda| \prod_{k=1}^{n}\left[|\xi||\lambda|^{-\frac{1}{l}}\right]^{\alpha_{k}}\|D(\xi, \lambda)\|_{B(E)} .
$$

Since $A$ is uniformly positive and $L(\xi) \in S_{\varphi_{1}}$, setting $y_{k}=\left(|\lambda|^{-\frac{1}{l}}\left|\xi_{k}\right|\right)^{\alpha_{k}}$ in the following wellknown inequality:

$$
y_{1}^{\alpha_{1}} y_{2}^{\alpha_{2}} \cdots y_{n}^{\alpha_{n}} \leq C\left(1+\sum_{k=1}^{n} y_{k}^{l}\right), \quad y_{k} \geq 0,|\alpha| \leq l,
$$

we obtain

$$
\left\|\sigma_{2}(\xi, \lambda)\right\|_{B(E)} \leq C \sum_{|\alpha| \leq l}|\lambda|\left[1+\sum_{k=1}^{n}\left|\xi_{k}\right|^{l}|\lambda|^{-1}\right]|\lambda+L(\xi)|^{-1} .
$$

Taking into account the Condition 2.1 and (2.1)-(2.3) we get

$$
\left\|\sigma_{2}(\xi, \lambda)\right\|_{B(E)} \leq C\left(|\lambda|+\sum_{k=1}^{n}\left|\xi_{k}\right|^{l}\right)(|\lambda|+|L(\xi)|)^{-1} \leq C .
$$


Lemma 2.2 Assume the Condition 2.1 holds. Suppose $\hat{a}_{\alpha} \in C^{(n)}\left(R^{n}\right),\left[D^{\beta} \hat{A}(\xi)\right] \hat{A}^{-1}\left(\xi_{0}\right) \in$ $C\left(R^{n} ; B(E)\right)$ and

$$
\begin{aligned}
& |\xi|^{|\beta|}\left|D^{\beta} a_{\alpha}(\xi)\right| \leq C_{1}, \quad \beta_{k} \in\{0,1\}, \xi \in R^{n} \backslash\{0\}, 0 \leq|\beta| \leq n, \\
& \left\||\xi|^{|\beta|}\left[D^{\beta} \hat{A}(\xi)\right] \hat{A}^{-1}\left(\xi_{0}\right)\right\|_{B(E)} \leq C_{2}, \quad \beta_{k} \in\{0,1\}, \xi, \xi_{0} \in R^{n} \backslash\{0\} .
\end{aligned}
$$

Then the operator functions $|\xi|^{|\beta|} D^{\beta} \sigma_{i}(\xi, \lambda), i=0,1,2$, are uniformly bounded.

Proof Let us first prove that $\xi_{k} \frac{\partial \sigma_{1}}{\partial \xi_{k}}$ is uniformly bounded. In fact,

$$
\left\|\xi_{k} \frac{\partial \sigma_{1}}{\partial \xi_{k}}\right\|_{B(E)} \leq\left\|I_{1}\right\|_{B(E)}+\left\|I_{2}\right\|_{B(E)}+\left\|I_{3}\right\|_{B(E)}
$$

where

$$
I_{1}=\left[\xi_{k} \frac{\partial \hat{A}(\xi)}{\partial \xi_{k}}\right] D(\xi, \lambda), \quad I_{2}=\hat{A}(\xi)\left[\xi_{k} \frac{\partial \hat{A}(\xi)}{\partial \xi_{k}}\right] D^{2}(\xi, \lambda)
$$

and

$$
I_{3}=\hat{A}(\xi)\left[\xi_{k} \frac{\partial L(\xi)}{\partial \xi_{k}}\right] D^{2}(\xi, \lambda)
$$

By using (2.3) and (2.5) we get

$$
\left\|I_{1}\right\|_{B(E)} \leq\left\|\left[\xi_{k} \frac{\partial \hat{A}(\xi)}{\partial \xi_{k}}\right] \hat{A}^{-1}\left(\xi_{0}\right)\right\|_{B(E)}\left\|\sigma_{1}\right\|_{B(E)} \leq C .
$$

Due to the positivity of $\hat{A}$, by using (2.3) and (2.5) we obtain

$$
\left\|I_{2}\right\|_{B(E)} \leq\left\|\left[\xi_{k} \frac{\partial \hat{A}(\xi)}{\partial \xi_{k}}\right] \hat{A}^{-1}\left(\xi_{0}\right)\right\|_{B(E)}\left\|\sigma_{1}\right\|_{B(E)}^{2} \leq C .
$$

Since $\hat{A}(\xi)$ is uniformly positive, by using (2.1), (2.3), and (2.5) for $\lambda \in S\left(\varphi_{2}\right)$ and $\varphi_{1}+\varphi_{2}<$ $\pi$ we get

$$
\left\|I_{3}\right\|_{B(E)} \leq\left|\xi_{k} \frac{\partial L}{\partial \xi_{k}}\right|\|D(\xi, \lambda)\|_{B(E)}\left\|\sigma_{1}(\xi, \lambda)\right\|_{B(E)} \leq C .
$$

In a similar way, the uniform boundedness of $\sigma_{0}(\xi, \lambda)$ is proved. Next we shall prove $\xi_{k} \frac{\partial \sigma_{2}}{\partial \xi_{k}}$ is uniformly bounded. Similarly,

$$
\left\|\xi_{k} \frac{\partial \sigma_{2}}{\partial \xi_{k}}\right\|_{B(E)} \leq\left\|J_{1}\right\|_{B(E)}+\left\|J_{2}\right\|_{B(E)},
$$

where

$$
J_{1}=\sum_{|\alpha| \leq l}|\lambda|^{1-\frac{|\alpha|}{l}}\left(\xi_{k} \frac{\partial a_{\alpha}}{\partial \xi_{k}}\right)\left[(i \xi)^{\alpha}+a_{\alpha}(\xi) i \alpha_{k}(i \xi)^{\alpha}\right] D(\xi, \lambda),
$$




$$
J_{2}=\sum_{|\alpha| \leq l}|\lambda|^{1-\frac{|\alpha|}{l}} a_{\alpha}(\xi)(i \xi)^{\alpha}\left[\xi_{k} \frac{\partial a_{\alpha}}{\partial \xi_{k}}+a_{\alpha}(\xi)(i \xi)^{\alpha}+\xi_{k} \frac{\partial \hat{A}(\xi)}{\partial \xi_{k}}\right] D^{2}(\xi, \lambda)
$$

First of all, we show $J_{1}$ is uniformly bounded. Since

$$
\left\|J_{1}\right\|_{B(E)} \leq \sum_{|\alpha| \leq l}\left|\xi_{k} \frac{\partial a_{\alpha}}{\partial \xi_{k}}\right|\left\|\xi^{\alpha}|\lambda|^{1-\frac{|\alpha|}{l}} D(\xi, \lambda)\right\|_{B(E)}
$$

due to positivity of $\hat{A}$, by virtue of (2.1) and (2.3)-(2.5) we obtain $\left\|J_{1}\right\|_{B(E)} \leq C$. In a similar way we have $\left\|J_{2}\right\|_{B(E)} \leq C$. Hence, the operator functions $\xi_{k} \frac{\partial \sigma_{i}}{\partial \xi_{k}}, i=0,1,2$ are uniformly bounded. From the representations of $\sigma_{i}(\xi, \lambda)$ it easy to see that operator functions $|\xi|^{\beta} D^{|\beta|} \sigma_{i}(\xi, \lambda)$ contain similar terms to $I_{k}$; namely, the functions $|\xi|^{|\beta|} D^{\beta} \sigma_{i}(\xi, \lambda)$ will be represented as combinations of principal terms

$$
\begin{aligned}
& \xi^{\mu}\left[D_{\xi}^{v} \hat{A}(\xi)+D_{\xi}^{v} a_{\alpha}(\xi)\right][D(\xi, \lambda)]^{|\beta|}, \\
& \sum_{|\alpha| \leq l}|\lambda|^{1-\frac{|\alpha|}{l}} \xi^{\mu} D_{\xi}^{v}\left[\hat{A}(\xi)+a_{\alpha}(\xi)\right][D(\xi, \lambda)]^{|\beta|},
\end{aligned}
$$

where $\mu, v$ are $n$-dimensional integers vectors and $|\mu|+|\nu| \leq|\beta|$. Therefore, by using similar arguments to the above and in view of (2.6) one can easily check that

$$
|\xi|^{|\beta|}\left\|D^{\beta} \sigma_{i}(\xi, \lambda)\right\|_{B(E)} \leq C, \quad i=0,1,2
$$

From [10] we obtain the following.

Lemma 2.3 Let all conditions of Lemma 2.2 hold. Suppose E is a Banach space satisfying the uniform multiplier condition. Then the sets

$$
\begin{aligned}
& S_{0}(\xi, \lambda)=\left\{|\xi|^{|\beta|} D_{\xi}^{\beta} \sigma_{0}(\xi, \lambda) ; \xi \in R^{n} \backslash\{0\}\right\}, \\
& S_{1}(\xi, \lambda)=\left\{|\xi|^{|\beta|} D_{\xi}^{\beta} \sigma_{1}(\xi, \lambda) ; \xi \in R^{n} \backslash\{0\}\right\}, \\
& S_{2}(\xi, \lambda)=\left\{|\xi|^{|\beta|} D_{\xi}^{\beta} \sigma_{2}(\xi, \lambda) ; \xi \in R^{n} \backslash\{0\}\right\}
\end{aligned}
$$

are uniformly $R$-bounded for $\beta_{k} \in\{0,1\}$ and $0 \leq|\beta| \leq n$.

Result 2.1 Suppose all conditions of the Lemma 2.2 are satisfied, $E$ is a $U M D$ space. Then the sets $S_{i}(\xi, \lambda), i=0,1,2$, are uniformly $R$-bounded.

Now we are ready to present our main results. We find sufficient conditions that guarantee the separability of problem (1.2).

Condition 2.2 Suppose the following are satisfied:

(1)

$$
L(\xi)=\sum_{|\alpha| \leq l} \hat{a}_{\alpha}(\xi)(i \xi)^{\alpha} \in S_{\varphi_{1}}, \quad \varphi_{1} \in[0, \pi), \quad|L(\xi)| \geq C \sum_{k=1}^{n}\left|\hat{a}_{k}\right|\left|\xi_{k}\right|^{l}, \quad \xi \in R^{n}
$$


(2) $\hat{a}_{\alpha} \in C^{(n)}\left(R^{n}\right)$ and

$$
|\xi|^{|\beta|}\left|D^{\beta} \hat{a}_{\alpha}(\xi)\right| \leq C_{1}, \quad \beta_{k} \in\{0,1\}, 0 \leq|\beta| \leq n
$$

(3) for $0 \leq|\beta| \leq n$,

$$
\begin{aligned}
& {\left[D^{\beta} \hat{A}(\xi)\right] \hat{A}^{-1}\left(\xi_{0}\right) \in C\left(R^{n} ; B(E)\right), \quad|\xi|^{|\beta|}\left\|\left[D^{\beta} \hat{A}(\xi)\right] \hat{A}^{-1}\left(\xi_{0}\right)\right\|_{B(E)} \leq C_{2},} \\
& \quad \xi, \xi_{0} \in R^{n} \backslash\{0\} .
\end{aligned}
$$

Let

$$
X=L_{p, \gamma}\left(R^{n} ; E\right), \quad Y=W_{p, \gamma}^{l}\left(R^{n} ; E(A), E\right), \quad p \in(1, \infty) .
$$

Theorem 2.1 Suppose the Condition 2.2 holds. Assume E is a Banach space satisfying the weighted multiplier condition. Let $\hat{A}$ be a uniformly $R$-positive in $E$ and $\lambda \in S_{\varphi_{2}}$ with $0 \leq \varphi_{A}+\varphi_{1}+\varphi_{2}<\pi$. Then the problem (1.2) has a unique solution $u$ and the coercive uniform estimate holds

$$
\sum_{|\alpha| \leq l}|\lambda|^{1-\frac{|\alpha|}{l}}\left\|a_{\alpha} * D^{\alpha} u\right\|_{X}+\|A * u\|_{X}+|\lambda|\|u\|_{X} \leq C\|f\|_{X}
$$

for all $f \in X$ and $\lambda \in S_{\varphi}$.

Proof By applying the Fourier transform to equation (1.2) we get

$$
\hat{u}(\xi)=D(\xi, \lambda) \hat{f}(\xi), \quad D(\xi, \lambda)=[\hat{A}(\xi)+L(\xi)+\lambda]^{-1}, \quad L(\xi)=\sum_{|\alpha| \leq l} \hat{a}_{\alpha}(\xi)(i \xi)^{\alpha}
$$

Hence, the solution of (1.2) can be represented as $u(x)=F^{-1} D(\xi, \lambda) \hat{f}$ and there are positive constants $C_{1}$ and $C_{2}$ so that

$$
\begin{aligned}
& C_{1}|\lambda|\|u\|_{X} \leq\left\|F^{-1}\left[\sigma_{0}(\xi, \lambda) \hat{f}\right]\right\|_{X} \leq C_{2}|\lambda|\|u\|_{X}, \\
& C_{1}\|A * u\|_{X} \leq\left\|F^{-1}\left[\sigma_{1}(\xi, \lambda) \hat{f}\right]\right\|_{X} \leq C_{2}\|A * u\|_{X}, \\
& C_{1} \sum_{|\alpha| \leq l}|\lambda|^{1-\frac{|\alpha|}{l}}\left\|a_{\alpha} * D^{\alpha} u\right\|_{X} \\
& \quad \leq\left\|F^{-1}\left[\sigma_{2}(\xi, \lambda) \hat{f}\right]\right\|_{X} \leq C_{2} \sum_{|\alpha| \leq l}|\lambda|^{1-\frac{|\alpha|}{l}}\left\|a_{\alpha} * D^{\alpha} u\right\|_{X},
\end{aligned}
$$

where $\sigma_{i}(\xi, \lambda)$ are operator functions defined in Lemma 2.1. Therefore, it is sufficient to show that the operator functions $\sigma_{i}(\xi, \lambda)$ are multipliers in $X$. However, this follows from Lemma 2.3. Thus, from (2.8) we obtain

$$
\begin{aligned}
& |\lambda|\|u\|_{X} \leq C_{0}\|f\|_{X}, \quad\|A * u\|_{X} \leq C_{1}\|f\|_{X}, \\
& \sum_{|\alpha| \leq l}|\lambda|^{1-\frac{|\alpha|}{l}}\left\|a_{\alpha} * D^{\alpha} u\right\|_{X} \leq C_{2}\|f\|_{X}
\end{aligned}
$$

for all $f \in X$. Hence, we get the assertion. 
Let $O$ be an operator in $X$ generated by problem (1.2) for $\lambda=0$, i.e.

$$
D(O) \subset Y, \quad O u=\sum_{|\alpha| \leq l} a_{\alpha} * D^{\alpha}+A * u .
$$

Result 2.2 Theorem 2.1 implies that the operator $O$ is separable in $X$, i.e. for all $f \in X$ there is a unique solution $u \in Y$ of the problem (1.2), all terms of equation (1.2) also are from $X$ and there are positive constants $C_{1}$ and $C_{2}$ so that

$$
C_{1}\|O u\|_{X} \leq \sum_{|\alpha| \leq l}\left\|a_{\alpha} * D^{\alpha} u\right\|_{X}+\|A * u\|_{X} \leq C_{2}\|O u\|_{X}
$$

Condition 2.3 Let $D(A(x))=D(\hat{A}(\xi)), D(\hat{A}(\xi))$ is dense in $E$ and does not depend on $\xi$; $A(x)$ is uniformly positive in $E$. Moreover, there are positive constants $C_{1}$ and $C_{2}$ so that, for $u \in D(A), x \in R^{n}$,

$$
C_{1}\left\|\hat{A}\left(\xi_{0}\right) u\right\| \leq\|A(x) u\| \leq C_{2}\left\|\hat{A}\left(\xi_{0}\right) u\right\| .
$$

Remark 2.1 The Condition 2.3 is checked for the regular elliptic operators with smooth coefficients on sufficiently smooth domains $\Omega \subset R^{m}$ considered in the Banach space $E=$ $L_{p_{1}}(\Omega), p_{1} \in(1, \infty)$ (see Theorem 4.1).

Theorem 2.2 Assume all conditions of Theorem 2.1 and Condition 2.3 are satisfied. Then for $f \in X$ and $\lambda \in S(\varphi)$ problem (1.2) has a unique solution $u \in Y$ and the coercive uniform estimate holds,

$$
\sum_{|\alpha| \leq l}|\lambda|^{1-\frac{|\alpha|}{l}}\left\|D^{\alpha} u\right\|_{X}+\|A u\|_{X} \leq C\|f\|_{X}
$$

Proof By applying the Fourier transform to equation (1.2) we obtain $D(\xi, \lambda) \hat{u}(\xi)=\hat{f}(\xi)$, where

$$
D(\xi, \lambda)=[\hat{A}(\xi)+L(\xi)+\lambda]^{-1}
$$

So, we see that the solution of equation (1.2) can be represented as $u(x)=F^{-1} D(\xi, \lambda) \hat{f}$. Moreover, by the Condition 2.3 we have

$$
\left\|A F^{-1} D(\xi, \lambda) \hat{f}\right\|_{X} \leq M\left\|\hat{A}\left(\xi_{0}\right) F^{-1} D(\xi, \lambda) \hat{f}\right\|_{X} .
$$

Hence, by using the estimates (2.8) it is sufficient to show that the operator functions $\sum_{|\alpha| \leq l}|\lambda|^{1-\frac{|\alpha|}{l}} \xi^{\alpha} D(\xi, \lambda)$ and $\hat{A}\left(\xi_{0}\right) D(\xi, \lambda)$ are multipliers in $X$. In fact, in view of (3) part of Condition 2.2 and $R$-positivity of $\hat{A}$ these are proved by reasoning as in Lemma 2.3.

Condition 2.4 Let the Condition 2.3 hold and let there be positive constants $C_{1}$ and $C_{2}$ such that

$$
C_{1} \sum_{k=1}^{n}\left|\hat{a}_{k}\right|\left|\xi_{k}\right|^{l} \leq|L(\xi)| \leq C_{2} \sum_{k=1}^{n}\left|\hat{a}_{k}\right|\left|\xi_{k}\right|^{l}, \quad \xi \in R^{n},
$$


and

$$
\begin{aligned}
& \hat{A}(\xi) A^{-1}\left(x_{0}\right) \in L_{\infty}\left(R^{n} ; B(E)\right), \quad \xi, x_{0} \in R^{n}, \\
& C_{1}\left\|A\left(x_{0}\right) u\right\| \leq\|A(x) u\| \leq C_{2}\left\|A\left(x_{0}\right) u\right\|, \quad u \in D(A), x \in R^{n} .
\end{aligned}
$$

Theorem 2.3 Assume all conditions of Theorem 2.2 and Condition 2.4 are satisfied. Then for $u \in Y$ there are positive constants $M_{1}$ and $M_{2}$ so that

$$
M_{1}\|u\|_{Y} \leq \sum_{|\alpha| \leq l}\left\|a_{\alpha} * D^{\alpha} u\right\|_{X}+\|A * u\|_{X} \leq M_{2}\|u\|_{Y}
$$

Proof The left part of the above inequality is derived from Theorem 2.2. So, it remains to prove the right side of the estimate. In fact, from Condition 2.4 for $u \in Y$ we have

$$
\begin{aligned}
\|A * u\|_{X} & \leq M\left\|F^{-1} \hat{A} \hat{u}\right\|_{X} \leq C\left\|F^{-1} \hat{A} A^{-1}\left(x_{0}\right) A\left(x_{0}\right) \hat{u}\right\|_{X} \\
& \leq C\left\|F^{-1} A\left(x_{0}\right) \hat{u}\right\|_{X} \leq C\|A u\|_{X} .
\end{aligned}
$$

Hence, applying the Fourier transform to equation (1.2) and by a reasoning as in Theorem 2.2, it is sufficient to prove that the function $\sum_{|\alpha| \leq l} \hat{a}_{\alpha} \xi^{\alpha}\left(\sum_{k=1}^{n} \xi_{k}^{l_{k}}\right)^{-1}$ is a uniform multiplier in $X$. In fact, by using the Condition 2.4 and the proof of Lemma 2.3 we get the desired result. Consider the following example.

Example 1 Let $m=2, n=2, E=\mathbb{C}, a_{\alpha}=a(x, y), A=b(x, y)$ such that $\hat{a}(\xi)$ and $\hat{b}(\xi)$ are positive real-valued functions for all $\xi \in R^{2}$ satisfying the Condition 2.2. Consider the equation

$$
\left[-a * \frac{\partial^{2} u}{\partial x^{2}}-a * \frac{\partial^{2} u}{\partial x \partial y}-a * \frac{\partial^{2} u}{\partial y^{2}}\right]+b * u=f(x, y)
$$

It is clear that the above equation satisfies all conditions of Theorem 2.1, i.e. the above problem is $L_{p}\left(R^{2}\right)$ separable.

Result 2.3 Theorems 2.3 implies that for all $u \in Y$ there are positive constants $C_{1}$ and $C_{2}$ so that

$$
C_{1}\|u\|_{Y} \leq\|O u\|_{X} \leq C_{2}\|u\|_{Y} .
$$

From Theorem 2.1 we have the following.

Result 2.4 Assume all conditions of Theorem 2.1 hold. Then, for all $\lambda \in S_{\varphi}$, the resolvent of operator $O$ exists and the following sharp estimate holds:

$$
\begin{aligned}
& \sum_{|\alpha| \leq l}|\lambda|^{1-\frac{|\alpha|}{l}}\left\|a_{\alpha} * D^{\alpha}(O+\lambda)^{-1}\right\|_{B(X)}+\left\|A *(O+\lambda)^{-1}\right\|_{B(X)} \\
& \quad+\left\|\lambda(O+\lambda)^{-1}\right\|_{B(X)} \leq C .
\end{aligned}
$$


Result 2.5 Theorem 2.1 particularly implies that the operator $O$ is uniformly sectorial in $X$; i.e. if $\hat{A}$ is uniformly $R$-positive for $\varphi \in\left(\frac{\pi}{2}, \pi\right)$ then (see e.g. [29], Section 1.14.5) the operator $O+a$ is a generator of an analytic semigroup in $X$.

From Theorems 2.1-2.3 and Proposition $\mathrm{A}_{0}$ we obtain the following.

Result 2.6 Let conditions of Theorems 2.1-2.3 hold for Banach spaces $E \in U M D$, respectively. Then assertions of Theorems 2.1-2.3 are valid.

\section{The Cauchy problem for parabolic CDOE}

In this section, we shall consider the following Cauchy problem for the convolution parabolic equation:

$$
\frac{\partial u}{\partial t}+\sum_{|\alpha| \leq l} a_{\alpha} * D^{\alpha} u+A * u=f(t, x), \quad u(0, x)=0
$$

where $A=A(x)$ is a possibly unbounded operator in a Banach space $E, a_{\alpha}=a_{\alpha}(x)$ are complex-valued functions. For $R_{+}^{n+1}, \mathbf{p}=\left(p, p_{1}\right), Z=L_{\mathbf{p}, \gamma}\left(R_{+}^{n+1} ; E\right)$ will denote the space of all $\mathbf{p}$-summable $E$-valued functions with mixed norm (see e.g. [26], Section 4, for the complex-valued case), i.e., the space of all measurable $E$-valued functions $f$ defined on $R_{+}^{n+1}$, for which

$$
\|f\|_{Z}=\left(\int_{R^{n}}\left(\int_{R_{+}}\|f(x, t)\|_{E}^{p} \gamma(x) d x\right)^{\frac{p_{1}}{p}} d t\right)^{\frac{1}{p_{1}}}<\infty .
$$

Let $E_{0}$ and $E$ be two Banach spaces, where $E_{0}$ continuously and densely embeds into $E$. Suppose $l$ is an integer and $W_{\mathbf{p}, \gamma}^{1, l}\left(R_{+}^{n+1} ; E_{0}, E\right)$ denotes the space of all functions $u \in Y$ such that we have the generalized derivatives $D_{t} u, D_{k}^{l} u \in Z$, with the norm

$$
\|u\|_{W_{\mathbf{p}, \gamma}^{1, l}\left(R_{+}^{n+1} ; E_{0}, E\right)}=\|u\|_{Z}+\left\|D_{y} u\right\|_{Z}+\sum_{k=1}^{n}\left\|D_{k}^{l} u\right\|_{Z} .
$$

Applying Theorem 2.1 we establish the maximal regularity of the problem (3.1) in the mixed norm $Z$. To this aim we need the following result.

Theorem 3.1 Suppose the Condition 2.2 holds, E is a Banach space that satisfies the uniform multiplier condition, and the operator $\hat{A}(\xi)$ is uniformly R-positive in $E$. Then the operator $O$ is uniformly R-positive in $X$.

Proof Result 2.3 implies that the operator $O$ is positive in $X$. We have to prove the $R$ boundedness of the set

$$
\sigma(\xi, \lambda)=\left\{\lambda(O+\lambda)^{-1}: \lambda \in S_{\varphi}\right\} .
$$

From the proof of Theorem 2.1 we have

$$
\lambda(O+\lambda)^{-1} f=F^{-1} \Phi(\xi, \lambda) \hat{f}, \quad f \in X,
$$


where

$$
\Phi(\xi, \lambda)=\lambda[\hat{A}(\xi)+L(\xi)+\lambda]^{-1}
$$

By definition of $R$-boundedness, it is enough to show that the operator function $\Phi(\xi, \lambda)$ (dependent on the variable $\lambda$ and the parameter $\xi$ ) is a multiplier in $X$. In a similar manner to Lemma 2.3 one can easily show that $\Phi(\xi, \lambda)$ is a multiplier in $X$. Then by the definition of $R$-boundedness we have

$$
\begin{aligned}
& \int_{0}^{1}\left\|\sum_{j=1}^{m} r_{j}(y) \lambda_{j}\left(O+\lambda_{j}\right)^{-1} f_{j}\right\|_{X} d y \\
& \quad=\int_{0}^{1}\left\|\sum_{j=1}^{m} r_{j}(y) F^{-1} \Phi\left(\xi, \lambda_{j}\right) \hat{f}_{j}\right\|_{X} d y \\
& =\int_{0}^{1}\left\|F^{-1} \sum_{j=1}^{m} r_{j}(y) \Phi\left(\xi, \lambda_{j}\right) \hat{f}_{j}\right\|_{X} d y \leq C \int_{0}^{1}\left\|\sum_{j=1}^{m} r_{j}(y) f_{j}\right\|_{X} d y
\end{aligned}
$$

for all $\xi_{1}, \xi_{2}, \ldots, \xi_{m} \in R^{n}, \lambda_{1}, \lambda_{2}, \ldots, \lambda_{m} \in S_{\varphi}, f_{1}, f_{2}, \ldots, f_{m} \in X, m \in \mathbb{N}$, where $\left\{r_{j}\right\}$ is a sequence of independent symmetric $\{-1,1\}$-valued random variables on $[0,1]$. Hence, the set $\sigma(\xi, \lambda)$ is uniformly $R$-bounded.

From Theorem 3.1 and Proposition $\mathrm{A}_{0}$ we obtain the following.

Result 3.1 Let conditions of Theorem 3.1 hold for the Banach spaces $E \in U M D$. Then the assertion of Theorem 3.1 is valid.

Now we are ready to state the main result of this section.

Theorem 3.2 Assume the Condition 2.2 holds for $\varphi \in\left(\frac{\pi}{2}, \pi\right), E \in U M D$, and the operator $\hat{A}(\xi)$ is uniformly $R$-positive in $E$. Then for all $f \in Z$ equation (3.1) has a unique solution $u \in W_{\mathbf{p}}^{1, l}\left(R_{+}^{n+1} ; E(A), E\right)$ satisfying

$$
\left\|\frac{\partial u}{\partial t}\right\|_{Z}+\sum_{|\alpha| \leq l}\left\|a_{\alpha} * D^{\alpha} u\right\|_{Z}+\|A * u\|_{Z} \leq C\|f\|_{Z}
$$

Proof It is clear that

$$
Z=L_{p_{1}}\left(R_{+} ; X\right), \quad W_{\mathbf{p}, \gamma}^{1, l}\left(R_{+}^{n+1} ; E(A), E\right)=W_{p_{1}}^{1}\left(R_{+} ; D(O), X\right)
$$

Therefore, the problem (3.1) can be expressed as

$$
\frac{d u}{d t}+O u(t)=f(t), \quad u(0)=0, \quad t \in R_{+} .
$$

By virtue of [1], Theorem 4.5.2, $X \in U M D$ provided $E \in U M D, p \in(1, \infty)$. Then due to $R$-positivity of $O$ with $\varphi \in\left(\frac{\pi}{2}, \pi\right)$, by virtue of [1], Proposition 8.10, we see that for $f \in$ 


$$
\begin{aligned}
& L_{p_{1}}\left(R_{+} ; X\right) \text { equation (3.3) has a unique solution } u \in W_{p_{1}}^{1}\left(R_{+} ; D(O), X\right) \text { satisfying } \\
& \qquad\left\|\frac{d u}{d t}\right\|_{L_{p_{1}}\left(R_{+} ; X\right)}+\|O u\|_{L_{p_{1}}\left(R_{+} ; X\right)} \leq C\|f\|_{L_{p_{1}}\left(R_{+} ; X\right) .} .
\end{aligned}
$$

In view of Results 2.2, 2.6, from the above estimate we get (3.2).

\section{Degenerate convolution-elliptic equations}

Consider the problem (1.1). Let

$$
\tilde{X}=L_{p}\left(R^{n} ; E\right), \quad \tilde{Y}=W_{p, \gamma}^{[l]}\left(R^{n} ; E(A), E\right), \quad p \in(1, \infty) .
$$

We show in this section the following result.

Theorem 4.1 Suppose the Condition 2.3 holds. Then for all $f \in \tilde{X}$ there is a unique solution of the problem (1.1) and the following coercive uniform estimate holds:

$$
\sum_{|\alpha| \leq l}|\lambda|^{1-\frac{|\alpha|}{l}}\left\|a_{\alpha} * D^{[\alpha]} u\right\|_{\tilde{X}}+\|A * u\|_{\tilde{X}}+|\lambda|\|u\|_{\tilde{X}} \leq C\|f\|_{\tilde{X}}
$$

Proof Let us make the following substitution:

$$
y_{k}=\int_{0}^{x_{k}} \gamma^{-1}(z) d z, \quad k=1,2, \ldots, n
$$

It is clear that under the substitution (4.2), $D^{[\alpha]} u$ transforms to $D^{\alpha} u$. Moreover, the spaces $\tilde{X}$ and $\tilde{Y}$ are mapped isomorphically onto the weighted spaces $L_{p, \tilde{\gamma}}\left(R^{n} ; E\right)$ and $W_{p, \tilde{\gamma}}^{l}\left(R^{n} ; E(A), E\right)$, respectively, where

$$
\gamma=\tilde{\gamma}(y)=\gamma(x(y))=\gamma\left(x_{1}\left(y_{1}\right), x_{2}\left(y_{2}\right), \ldots, x_{n}\left(y_{n}\right)\right) .
$$

Moreover, under the substitution (4.2) the degenerate problem (1.1) is transformed into the following non-degenerate problem:

$$
\sum_{|\alpha| \leq l} a_{\alpha} * D^{\alpha} u+(A+\lambda) * u=f
$$

considered in the weighted space $L_{p, \widetilde{\gamma}}\left(R^{n} ; E\right)$ where

$$
a_{\alpha}=a_{\alpha}(\widetilde{\gamma}(y)), \quad u=\tilde{u}(y)=u(\widetilde{\gamma}(y)), \quad A=\tilde{A}(y)=A(\widetilde{\gamma}(y)), \quad f=\tilde{f}(y)=f(\widetilde{\gamma}(y)) .
$$

Then in view of Theorem 2.1 we obtain the assertion.

Now, we consider the Cauchy problem the degenerate parabolic convolution equation

$$
\begin{aligned}
& \frac{\partial u}{\partial t}+\sum_{|\alpha| \leq l} a_{\alpha} * D^{[\alpha]} u+A * u=f(t, x), \\
& u(0, x)=0, \quad t \in \mathbb{R}_{+}, x \in R^{n} .
\end{aligned}
$$

By using the map (4.2) we derive from Theorem 3.2 the following result. 
Theorem 4.2 Assume the Condition 2.3 holds, for $\frac{\pi}{2}<\varphi<\pi$, then problem (4.4) for all $f \in L_{\mathbf{p}}\left(R_{+}^{n+1} ; E\right)\left(\mathbf{p}=\left(p, p_{1}\right)\right)$ has a unique solution $u(t, x)$ and the following estimate holds:

$$
\left\|\frac{\partial u}{\partial t}\right\|_{L_{\mathbf{p}}\left(R_{+}^{n+1} ; E\right)}+\sum_{|\alpha| \leq l}\left\|a_{\alpha} * D^{[\alpha]} u\right\|_{L_{\mathbf{p}}\left(R_{+}^{n+1} ; E\right)}+\|A * u\|_{L_{\mathbf{p}}\left(R_{+}^{n+1} ; E\right)} \leq C\|f\|_{L_{\mathbf{p}}\left(R_{+}^{n+1} ; E\right)} .
$$

Result 4.1 If we take $|\alpha|=2, n=2$, then we see from Theorem 4.2 that the Cauchy problem

$$
\frac{\partial u}{\partial t}+a_{11} * \frac{\partial^{[2]} u}{\partial x_{1}^{2}}+a_{12} * \frac{\partial^{[2]} u}{\partial x_{1} \partial x_{2}}+a_{22} * \frac{\partial^{[2]} u}{\partial x_{2}^{2}}+A * u=f(t, x), \quad u(0, x)=0,
$$

has a unique solution satisfying the coercive estimate

$$
\left\|\frac{\partial u}{\partial t}\right\|_{L_{\mathbf{p}}\left(R_{+}^{3} ; E\right)}+\sum_{i, j=1}^{2}\left\|a_{i j} * \frac{\partial^{[2]} u}{\partial x_{i}^{[1]} \partial x_{j}^{[1]}}\right\|_{L_{\mathbf{p}}\left(R_{+}^{3} ; E\right)}+\|A * u\|_{L_{\mathbf{p}}\left(R_{+}^{3} ; E\right)} \leq C\|f\|_{L_{\mathbf{p}}\left(R_{+}^{3} ; E\right)}
$$

for all $f \in L_{P}\left(R_{+}^{3} ; E\right)$.

\section{Boundary value problems for integro-differential equations}

In this section by applying Theorem 2.1, the BVP for the anisotropic type convolution equations is studied. The maximal regularity properties of this problem in weighted mixed $L_{\mathbf{p}, \gamma}$ norms are derived. In this direction we can mention e.g. [2, 16], and [19].

Let $\tilde{\Omega}=R^{n} \times \Omega$, where $\Omega \subset R^{\mu}$ is an open connected set with compact $C^{2 m}$-boundary $\partial \Omega$. Consider the BVP for integro-differential equation

$$
\begin{aligned}
& (L+\lambda) u=\sum_{|\alpha| \leq l} a_{\alpha} * D^{\alpha} u+\sum_{|\alpha| \leq 2 m}\left(b_{\alpha} \eta_{\alpha} D_{y}^{\alpha}+\lambda\right) * u=f(x, y), \quad x \in R^{n}, y \in \Omega, \\
& B_{j} u=\sum_{|\beta| \leq m_{j}} b_{j \beta}(y) D_{y}^{\beta} u(x, y)=0, \quad y \in \partial \Omega, j=1,2, \ldots, m,
\end{aligned}
$$

where

$$
\begin{aligned}
& D_{j}=-i \frac{\partial}{\partial y_{j}}, \quad y=\left(y_{1}, \ldots, y_{\mu}\right), \quad b_{\alpha}=b_{\alpha}(x), \quad \eta_{\alpha}=\eta_{\alpha}(y), \\
& a_{\alpha}=a_{\alpha}(x), \quad \alpha=\left(\alpha_{1}, \alpha_{2}, \ldots, \alpha_{n}\right), \quad a_{\alpha}=a_{\alpha}(x), \quad u=u(x, y) .
\end{aligned}
$$

In general, $l \neq 2 m$ so, equation (5.1) is anisotropic. For $l=2 m$ we get an isotropic equation. Let $\tilde{\Omega}=R^{n} \times \Omega, \mathbf{p}=\left(p_{1}, p\right)$, and $\gamma(x)=|x|^{\alpha}, L_{\mathbf{p}, \gamma}(\tilde{\Omega})$ will denote the space of all $\mathbf{p}$-summable scalar-valued functions with mixed norm (see e.g. [26], Section 4), i.e. the space of all measurable functions $f$ defined on $\tilde{\Omega}$, for which

$$
\|f\|_{L_{\mathbf{p}, \gamma}(\tilde{\Omega})}=\left(\int_{R^{n}}\left(\int_{\Omega}|f(x, y)|^{p_{1}} \gamma(x) d x\right)^{\frac{p}{p_{1}}} d y\right)^{\frac{1}{p}}<\infty .
$$

Analogously, $W_{\mathbf{p}, \gamma}^{l}(\tilde{\Omega})$ denotes the weighted Sobolev space with corresponding mixed norm [26], Section 10. Let $Q$ denote the operator generated by problem (5.1)-(5.2). In this section we present the following result. 
Theorem 5.1 Let the following conditions be satisfied:

(1) $\eta_{\alpha} \in C(\bar{\Omega})$ for each $|\alpha|=2 m$ and $\eta_{\alpha} \in L_{\infty}(\Omega)+L_{r_{k}}(\Omega)$ for each $|\alpha|=k<2 m$ with $r_{k} \geq p_{1}, p_{1} \in(1, \infty)$, and $2 m-k>\frac{l}{r_{k}}, v_{\alpha} \in L_{\infty},-1<\alpha<p-1, k=1,2, \ldots, n$;

(2) $b_{j \beta} \in C^{2 m-m_{j}}(\partial \Omega)$ for each $j, \beta, m_{j}<2 m, p \in(1, \infty), \lambda \in S_{\varphi}, \varphi \in[0, \pi)$;

(3) for $y \in \bar{\Omega}, \xi \in R^{\mu}, \sigma \in S_{\varphi_{0}}, \varphi_{0} \in\left(0, \frac{\pi}{2}\right),|\xi|+|\sigma| \neq 0$ let $\sigma+\sum_{|\alpha|=2 m} \eta_{\alpha}(y) \xi^{\alpha} \neq 0$;

(4) for each $y_{0} \in \partial \Omega$ local BVP in local coordinates corresponding to $y_{0}$

$$
\begin{aligned}
& \sigma+\sum_{|\alpha|=2 m} \eta_{\alpha}\left(y_{0}\right) D^{\alpha} \vartheta(y)=0, \\
& B_{j 0} \vartheta=\sum_{|\beta|=m_{j}} b_{j \beta}\left(y_{0}\right) D^{\beta} \vartheta(y)=h_{j}, \quad j=1,2, \ldots, m,
\end{aligned}
$$

has a unique solution $\vartheta \in C_{0}\left(R_{+}\right)$for all $h=\left(h_{1}, h_{2}, \ldots, h_{m}\right) \in R^{m}$ and for $\xi^{\prime} \in R^{\mu-1}$ with $\left|\xi^{\prime}\right|+|\lambda| \neq 0$

(5) the (1) part of Condition 2.2 is satisfied, $\hat{a}_{\alpha}, \hat{b}_{\alpha} \in C^{(n)}\left(R^{n}\right)$ and there are positive constants $C_{i}, i=1,2$, so that

$$
\begin{aligned}
& |\xi|^{|\beta|}\left|D^{\beta} \hat{a}_{\alpha}(\xi)\right| \leq C_{1}, \quad|\xi|^{|\beta|}\left|D^{\beta} \hat{b}_{\alpha}(\xi)\right| \leq C_{2}\left|\hat{b}_{\alpha}(\xi)\right| \\
& \xi \in R^{n} \backslash\{0\}, \beta_{k} \in\{0,1\}, 0 \leq|\beta| \leq n .
\end{aligned}
$$

Then for $f \in L_{\mathbf{p}, \gamma}(\tilde{\Omega})$ and $\lambda \in S_{\varphi}$ problem (5.1)-(5.2) has a unique solution $u \in W_{\mathbf{p}, \gamma}^{l}(\tilde{\Omega})$ and the following coercive uniform estimate holds:

$$
\begin{aligned}
& \sum_{|\alpha| \leq l}|\lambda|^{1-\frac{|\alpha|}{l}}\left\|a_{\alpha} * D^{\alpha} u\right\|_{L_{\mathbf{p}, \gamma}(\tilde{\Omega})}+\||\lambda| u\|_{L_{\mathbf{p}, \gamma}(\tilde{\Omega})} \\
& \quad+\sum_{|\alpha| \leq 2 m}\left\|b_{\alpha} \eta_{\alpha} D^{\alpha} * u\right\|_{L_{\mathbf{p}, \gamma}(\tilde{\Omega})} \leq C\|f\|_{L_{\mathbf{p}, \gamma}(\tilde{\Omega})} .
\end{aligned}
$$

Proof Let $E=L_{p_{1}}(\Omega)$. It is well known [27] that $L_{p_{1}}(\Omega)$ is a $U M D$ space for $p_{1} \in(1, \infty)$. Consider the operator $A$ in $L_{p_{1}}(\Omega)$ defined by

$$
D(A)=W_{p_{1}}^{2 m}\left(\Omega ; B_{j} u=0\right), \quad A(x) u=\sum_{|\alpha| \leq 2 m} b_{\alpha}(x) \eta_{\alpha}(y) D^{\alpha} u(y) .
$$

Therefore, the problem (5.1)-(5.2) can be rewritten in the form of (1.2), where $u(x)=$ $u(x, \cdot), f(x)=f(x, \cdot)$ are functions with values in $E=L_{p_{1}}(\Omega)$. It is easy to see that $\hat{A}(\xi)$ and $D^{\beta} \hat{A}(\xi)$ are operators in $L_{p_{1}}(\Omega)$ defined by

$$
\begin{aligned}
& D(\hat{A})=D\left(D^{\beta} \hat{A}\right)=W_{p_{1}}^{2 m}\left(\Omega ; B_{j} u=0\right), \quad \hat{A}(\xi) u=\sum_{|\alpha| \leq 2 m} \hat{b}_{\alpha}(\xi) \eta_{\alpha}(y) D^{\alpha} u(y), \\
& D_{\xi}^{\beta} \hat{A}(\xi) u=\sum_{|\alpha| \leq 2 m} D_{\xi}^{\beta} \hat{b}_{\alpha}(\xi) \eta_{\alpha}(y) D^{\alpha} u(y) .
\end{aligned}
$$

By virtue of [10], Theorem 4.1, we have

$$
\|(\hat{A}+\mu) u\|_{L_{p_{1}}(\Omega)} \leq C\|u\|_{W_{p_{1}}^{2 m}(\Omega)} \leq C\|(\hat{A}+\mu) u\|_{L_{p_{1}}(\Omega)},
$$




$$
\left\|\left(D^{\beta} \hat{A}+\mu\right) u\right\|_{L_{p_{1}}(\Omega)} \leq C\|u\|_{W_{p_{1}}^{2 m}(\Omega)} \leq C\left\|\left(D^{\beta} \hat{A}+\mu\right) u\right\|_{L_{p_{1}}(\Omega)} .
$$

Moreover, by using condition (5), for $u \in W_{p_{1}}^{2 m}(\Omega)$ we have

$$
|\xi|^{|\beta|}\left\|\left(D_{\xi}^{\beta} \hat{A}+\mu\right) u\right\|_{L_{p_{1}}(\Omega)} \leq C\|(\hat{A}+\mu) u\|_{L_{p_{1}}(\Omega)} .
$$

Moreover, by [28] we see that the space $L_{p_{1}}(\Omega)$ satisfies the multiplier condition. Then all conditions of Theorem 2.1 hold and we obtain the assertion.

\section{Infinite system of IDEs}

Consider the following infinite system of convolution equations:

$$
\sum_{|\alpha| \leq l} a_{\alpha} * D^{\alpha} u_{m}+\sum_{j=1}^{\infty}\left(d_{j}+\lambda\right) * u_{j}(x)=f_{m}(x), \quad x \in R^{n}, m=1,2, \ldots
$$

Condition 6.1 Let $-1<\alpha<p-1, k=1,2, \ldots, n$. There are positive constants $C_{1}$ and $C_{2}$ so that, for $\left\{d_{j}(x)\right\}_{1}^{\infty} \in l_{q}$, for all $x \in R^{n}$ and some $x_{0} \in R^{n}$,

$$
C_{1}\left|d_{j}\left(x_{0}\right)\right| \leq\left|d_{j}(x)\right| \leq C_{2}\left|d_{j}\left(x_{0}\right)\right| .
$$

Suppose $\hat{a}_{\alpha}, \hat{d}_{m} \in C^{(n)}\left(R^{n}\right)$ and there are positive constants $M_{i}, i=1,2$, so that

$$
\begin{aligned}
& |\xi|^{|\beta|}\left|D^{\beta} \hat{a}_{\alpha}(\xi)\right| \leq M_{1}, \quad|\xi|^{|\beta|}\left|D^{\beta} \hat{d}_{m}(\xi)\right| \leq M_{2}\left|\hat{d}_{m}(\xi)\right|, \\
& \xi \in R^{n} \backslash\{0\}, \beta_{k} \in\{0,1\}, 0 \leq|\beta| \leq n .
\end{aligned}
$$

Let

$$
\begin{aligned}
& D(x)=\left\{d_{m}(x)\right\}, \quad d_{m}>0, \quad u=\left\{u_{m}\right\}, \quad D * u=\left\{d_{m} * u_{m}\right\}, \\
& l_{q}(D)=\left\{u \in l_{q},\|u\|_{l_{q}(D)}=\left(\sum_{m=1}^{\infty}\left|d_{m}\left(x_{0}\right) * u_{m}\right|^{q}\right)^{\frac{1}{q}}<\infty\right\}, \quad 1<q<\infty .
\end{aligned}
$$

Here $\gamma(x)=|x|^{\alpha}$. Let $Q$ be a differential operator in $L_{p, \gamma}\left(R^{n} ; l_{q}\right)$ generated by problem (6.1) and

$$
B=B\left(L_{p, \gamma}\left(R^{n} ; l_{q}\right)\right) .
$$

Applying Theorem 2.1 we have the following.

Theorem 6.1 Suppose Condition 2.2(1) and Condition 6.1 are satisfied. Then:

(a) for all $f(x)=\left\{f_{m}(x)\right\}_{1}^{\infty} \in L_{p, \gamma}\left(R^{n} ; l_{q}(D)\right)$, for $\lambda \in S_{\varphi}, \varphi \in[0, \pi)$ problem (6.1) a unique solution $u=\left\{u_{m}(x)\right\}_{1}^{\infty}$ that belongs to $W_{p, \gamma}^{l}\left(R^{n} ; l_{q}(D), l_{q}\right)$ and the coercive uniform estimate holds,

$$
\begin{aligned}
& \sum_{|\alpha| \leq l}|\lambda|^{1-\frac{|\alpha|}{l}}\left\|a_{\alpha} * D^{\alpha} u\right\|_{L_{p, \gamma}\left(R^{n} ; l_{q}\right)}+\|D * u\|_{L_{p, \gamma}\left(R^{n} ; l_{q}\right)} \\
& \quad+|\lambda|\|u\|_{L_{p, \gamma}\left(R^{n} ; l_{q}\right)} \leq C\|f\|_{L_{p, \gamma}\left(R^{n} ; l_{q}\right)} ;
\end{aligned}
$$


(b) for $\lambda \in S_{\varphi}$ there exists a resolvent $(Q+\lambda)^{-1}$ and

$$
\begin{aligned}
& \sum_{|\alpha| \leq l}|\lambda|^{1-\frac{|\alpha|}{l}}\left\|a_{\alpha} *\left[D^{\alpha}(Q+\lambda)^{-1}\right]\right\|_{B} \\
& \quad+\left\|D *(Q+\lambda)^{-1}\right\|_{B}+\left\|\lambda(Q+\lambda)^{-1}\right\|_{B} \leq C .
\end{aligned}
$$

Proof In fact, let $E=l_{q}$ and $A=\left[d_{m}(x) \delta_{j m}\right], m, j=1,2, \ldots, \infty$. Then

$$
\hat{A}(\xi)=\left[\hat{d}_{m}(\xi) \delta_{j m}\right], \quad D^{\beta} \hat{A}(\xi)=\left[D^{\beta} \hat{d}_{m}(\xi) \delta_{j m}\right], \quad m, j=1,2, \ldots, \infty
$$

It is easy to see that $\hat{A}(\xi)$ is uniformly $R$-positive in $l_{q}$ and all conditions of Theorem 2.1 hold. Moreover, by [28] we see that the space $l_{q}$ satisfies the multiplier condition. Therefore, by virtue of Theorem 2.1 and Result 2.4 we obtain the assertions.

Remark 6.1 There are a lot of positive operators in concrete Banach spaces. Therefore, putting in (1.1) and (3.1) concrete Banach spaces instead of $E$ and concrete positive differential, pseudo differential operators, or finite, infinite matrices, etc. instead of $A$, by virtue of Theorem 2.1 and Theorem 3.2 we can obtain the maximal regularity properties of different classes of convolution equations and Cauchy problems for parabolic CDEs or their systems, respectively.

\section{Competing interests}

The authors declare that they have no competing interests.

\section{Authors' contributions}

All authors contributed equally to the writing of this paper. All authors read and approved the final manuscript.

\section{Author details}

${ }^{1}$ Baku State University, Baku, Azerbaijan. ${ }^{2}$ Department of Mechanical Engineering, Okan University, Akfirat Beldesi, Tuzla, Istanbul, 34959, Turkey. ${ }^{3}$ Department of Mathematics, Azerbaijan Khazar University, Baku, Azerbaijan.

Received: 14 December 2015 Accepted: 7 February 2016 Published online: 23 February 2016

\section{References}

1. Amann, H: Operator-valued Fourier multipliers, vector-valued Besov spaces, and applications. Math. Nachr. 186, 5-56 (1997)

2. Ashyralyev, A: On well-posedeness of the nonlocal boundary value problem for elliptic equations. Numer. Funct. Anal. Optim. 24(1-2), 1-15 (2003)

3. Agarwal, R, O'Regan, D, Shakhmurov, VB: Separable anisotropic differential operators in weighted abstract spaces and applications. J. Math. Anal. Appl. 338, 970-983 (2008)

4. Dore, G, Yakubov, S: Semigroup estimates and non coercive boundary value problems. Semigroup Forum 60, 93-121 (2000)

5. Denk, R, Hieber, M, Prüss, J: R-Boundedness, Fourier multipliers and problems of elliptic and parabolic type. Mem. Am. Math. Soc. 166, $788(2003)$

6. Haller, R, Heck, H, Noll, A: Mikhlin's theorem for operator-valued Fourier multipliers in $n$ variables. Math. Nachr. 244 110-130 (2002)

7. Krein, SG: Linear Differential Equations in Banach Space. Am. Math. Soc., Providence (1971)

8. Shakhmurov, VB, Shahmurov, R: Sectorial operators with convolution term. Math. Inequal. Appl. 13(2), 387-404 (2010)

9. Shakhmurov, VB, Musayev, H: Separability properties of convolution-differential operator equations in weighted $L_{p}$ spaces. Appl. Comput. Math. 14(2), 221-233 (2015)

10. Shakhmurov, VB: Separable convolution-elliptic operator with parameters. Forum Math. 27(6), 2637-2660 (2015)

11. Shakhmurov, VB: Maximal B-regular boundary value problems with parameters. J. Math. Anal. Appl. 320, 1-19 (2006)

12. Shakhmurov, VB: Embedding operators and maximal regular differential-operator equations in Banach-valued function spaces. J. Inequal. Appl. 2005(4), 329-345 (2005)

13. Weis, L: Operator-valued Fourier multiplier theorems and maximal $L_{p}$ regularity. Math. Ann. 319, 735-758 (2001)

14. Yakubov, S, Yakubov, Y: Differential-Operator Equations. Ordinary and Partial Differential Equations. Chapman \& Hall/CRC, Boca Raton (2000)

15. Clément, P, Gripenberg, G, Londen, S-O: Schauder estimates for equations with fractional derivatives. Trans. Am. Math. Soc. 352(5), 2239-2260 (2000) 
16. Zacher, R: Weak solutions of abstract evolutionary integro-differential equations in Hilbert spaces. Funkc. Ekvacioj 52(1), 1-18 (2009)

17. Keyantuo, V, Lizama, C: Maximal regularity for a class of integro-differential equations with infinite delay in Banach spaces. Stud. Math. 168, 25-50 (2005)

18. Prüss, J: Evolutionary Integral Equations and Applications. Birkhäuser, Basel (1993)

19. Poblete, V: Solutions of second-order integro-differential equations on periodic Besov spaces. Proc. Edinb. Math. Soc. 50, 477-492 (2007)

20. Arendt, W, Bu, S: Tools for maximal regularity. Math. Proc. Camb. Philos. Soc. 1, 317-336 (2003)

21. Girardi, $M$, Weis, L: Operator-valued multiplier theorems on $L_{p}(X)$ and geometry of Banach spaces. J. Funct. Anal. 204(2), 320-354 (2003)

22. Hytönen, T, Weis, L: Singular convolution integrals with operator-valued kernels. Math. Z. 255, 393-425 (2007)

23. Monniaux, S: On uniqueness for the Navier-Stokes system in 3D-bounded Lipschitz domains. J. Funct. Anal. 195, 1-11 (2002)

24. Dehghan, M, Shakeri, F: Solution of an integro-differential equation arising in oscillating magnetic fields using He's homotopy perturbation method. Prog. Electromagn. Res. 78, 361-378 (1978)

25. Amann, H: On the strong solvability of the Navier-Stokes equations. J. Math. Fluid Mech. 2, $16-98$ (2000)

26. Besov, OV, Ilin, VP, Nikolskii, SM: Integral Representations of Functions and Embedding Theorems. Nauka, Moscow (1975)

27. Burkholder, DL: A geometrical conditions that implies the existence certain singular integral of Banach space-valued functions. In: Proc. Conf. Harmonic Analysis in Honor of Antoni Zygmund, Chicago, 1981, pp. 270-286. Wadsworth, Belmont (1983)

28. Triebel, H: Spaces of distributions with weights. Multiplier on $L_{D}$ spaces with weights. Math. Nachr. 78, 339-356 (1977)

29. Triebel, H: Interpolation Theory, Function Spaces, Differential Operators. North-Holland, Amsterdam (1978)

\section{Submit your manuscript to a SpringerOpen ${ }^{\circ}$ journal and benefit from:}

- Convenient online submission

- Rigorous peer review

- Immediate publication on acceptance

- Open access: articles freely available online

- High visibility within the field

Retaining the copyright to your article 